\title{
Hippocampal efferents to retrosplenial cortex and lateral septum are required for memory acquisition
}

\author{
Ashley N. Opalka and Dong V. Wang \\ Department of Neurobiology and Anatomy, Drexel University College of Medicine, Philadelphia, Pennsylvania 19129, USA
}

\begin{abstract}
Learning and memory involves a large neural network of many brain regions, including the notable hippocampus along with the retrosplenial cortex (RSC) and lateral septum (LS). Previous studies have established that the dorsal hippocampus (dHPC) plays a critical role during the acquisition and retrieval/expression of episodic memories. However, the role of downstream circuitry from the dHPC, including the dHPC-to-RSC and dHPC-to-LS pathways, has come under scrutiny only recently. Here, we used an optogenetic approach with contextual fear conditioning in mice to determine whether the above two pathways are involved in acquisition and expression of contextual fear memory. We found that a selective inhibition of the AHPC neuronal terminals in either the RSC or LS during acquisition impaired subsequent memory performance, suggesting that both the dHPC-to-RSC and dHPC-to-LS pathways play a critical role in memory acquisition. We also selectively inhibited the two dHPC efferent pathways during memory retrieval and found a differential effect on memory performance. These results indicate the intricacies of memory processing and that hippocampal efferents to cortical and subcortical regions may be differentially involved in aspects of physiological and cognitive memory processes.
\end{abstract}

[Supplemental material is available for this article.]

Processing of memory entails multiple stages: acquisition, the initial encoding of memory; consolidation, the transformation of newly acquired information into long-lasting memory; retrieval, the recall of stored memory; and expression, the behavioral readout of recalled memory. Extensive research has shown that the dorsal hippocampus (dHPC) plays an important role in each of these memory processing stages (Scoville and Milner 1957; Maren and Fanselow 1997; McEchron et al. 1998; Lee and Kesner 2004; Misane et al. 2005; Carr et al. 2011; Goshen et al. 2011; Pierson et al. 2015; Ocampo et al. 2017). However, how the dHPC communicates with downstream cortical and subcortical regions during these memory stages remains unclear. Recent studies have shown that a number of cortical regions display increased activity during memory acquisition and expression stages (Takata et al. 2015; Miller et al. 2017; DeNardo et al. 2019) and that hippocampalcortical communications are required for memory acquisition and consolidation (Kitamura et al. 2017; DeNardo et al. 2019; Yamawaki et al. 2019a). Thus, recruitment of cortical brain regions during the initial acquisition stage may be necessary for subsequent consolidation and retrieval/expression of memories (Lesburguères et al. 2011; Kitamura et al. 2017; DeNardo et al. 2019; de Sousa et al. 2019). Additionally, recruitment of hippocampal projections to subcortical regions appears to be necessary during these memory processes as well (Olton et al. 1978; Hunsaker et al. 2009; Roy et al. 2017; Besnard et al. 2019, 2020).

The dHPC projects directly to a few cortical and subcortical regions, notably the granular retrosplenial cortex (RSC) and the lateral septum (LS) (Van Groen and Wyss 1990; Jinno et al. 2007; Miyashita and Rockland 2007; Kwapis et al. 2015; Takata et al. 2015; Todd and Bucci 2015). However, the function of these two dHPC projections during memory processes has come under scrutiny only recently (Yamawaki et al. 2019a,b; Besnard et al. 2020; Nitzan et al. 2020; Opalka et al. 2020). Previous studies revealed

\section{Corresponding author: dw657@drexel.edu}

Article is online at http://www.learnmem.org/cgi/doi/10.1101/lm.051797.120. that the RSC contributes to contextual memory (Keene and Bucci 2008; Corcoran et al. 2011; Cowansage et al. 2014; Kwapis et al. 2015; Robinson et al. 2018; de Sousa et al. 2019), spatial memory (Cooper et al. 2001; Czajkowski et al. 2014; Mao et al. 2017; Milczarek et al. 2018; Vann and Aggleton 2002), inhibitory avoidance memory (Katche et al. 2013; Katche and Medina 2017), and multisensory association (Robinson et al. 2011, 2014). Moreover, the RSC is one of few cortical regions that receive direct projections from the dHPC, positioning the RSC as a potential bridge in connecting the hippocampus with other cortical regions, such as the anterior cingulate cortex, medial prefrontal cortex, and secondary sensory cortices, to support long-term memory (Frankland et al. 2004; Maviel et al. 2004; Todd and Bucci 2015; Wang and Ikemoto 2016; Kitamura et al. 2017; DeNardo et al. 2019). Therefore, a better understanding of this dHPC-to-RSC connection will shed light on memory acquisition and retrieval/expression processes.

Further, previous literature reports the LS as essential for processing multiple forms of memory, such as contextual memory (Calandreau et al. 2007, 2010; Besnard et al. 2019; Besnard et al. 2020), social encounter and memory (Dantzer et al. 1988; Everts and Koolhaas 1997; Leroy et al. 2018), and addiction memory (McGlinchey and Aston-Jones 2018). Due to the hippocampalseptal involvement in theta oscillation (Colgin 2016), the dHPC-to-LS pathway has been recently investigated for its role in spatial navigation and spatial memory (Tingley and Buzsáki 2018). In particular, the firing of LS neurons phase-locked to dHPC theta oscillation (Mondragón-Rodríguez et al. 2019) positions this dHPC-to-LS pathway as a candidate for memory processing. To test whether the dHPC-to-RSC and dHPC-to-LS neural

\footnotetext{
C 2020 Opalka and Wang This article is distributed exclusively by Cold Spring Harbor Laboratory Press for the first 12 months after the full-issue publication date (see http://learnmem.cshlp.org/site/misc/terms.xhtml). After 12 months, it is available under a Creative Commons License (Attribution-NonCommercial 4.0 International), as described at http://creativecommons.org/licenses/by-nc/ $4.0 /$
} 
pathways are involved in the acquisition and retrieval/expression of memory, we used an optogenetic approach and contextual fear conditioning, a procedure widely used to assess hippocampusthe cortical (RSC) and subcortical (LS) projections from the dHPC are required during acquisition of the fear memory. These hippocampal efferents to downstream cortical and subcortical regions may process distinct features in representing a memory in its entirety.

\section{Results}

We bilaterally injected AAV viruses that encode fluorescent eYFP under the promoter CaMKII into the medial portion of the dHPC region (including medial CA1 and dorsal subiculum; Supplemental Figs. 1, 2). Consistent with previous work (Oh et al. 2014; Wyss and Van Groen 1992), our results showed that this dHPC region projected primarily to the granular RSC layer 3, midline LS, entorhinal cortex, and mammillary area. In the present study, we optogenetically inhibited two dHPC efferent pathways: the dHPC-to-RSC and dHPC-to-LS pathways (Supplemental Fig. 1). Repeated measures two-way ANOVAs were conducted on freezing behavior for each of the following experiments followed by Bonferroni post-hoc analyses. The between-subjects variables included two levels of treatment: halorhodopsin (Halo) or eYFP (Ctrl) viral injections. The within-subjects variables included two levels of time: recent (day 1) or remote (day 31 ) memory tests.

\section{Optogenetic inhibition of dHPC-to-RSC pathway during memory acquisition}

Mice received bilateral dHPC injections, targeting medial CA1 and rostral-dorsal subiculum (Supplemental Figs. 1, 2), of either AAV-Halo (Halo) or AAV-eYFP (Ctrl). Meanwhile, an optical fiber dependent memory. Our results provide direct evidence that both

was implanted in the midline of the RSC, capable of inhibiting dHPC projections from both hemispheres because the dHPC projects predominantly to RSC layer 3, located close to the midline (Fig. 1A, left; Supplemental Fig. 1). Four-five weeks after surgery, mice received photoinhibition of the dHPC-to-RSC neuronal terminals during acquisition of contextual fear memory on day 0 . Mice were tested subsequently for recent memory in $1 \mathrm{~d}$ and remote memory in $31 \mathrm{~d}$, receiving no photoinhibition during the tests (Fig. 1A, right). There was a significant main effect of viral group $\left(F_{(1,46)}=9.384, P=0.004 ;\right.$ repeated measures two-way ANOVA; Fig. 1B), indicating a substantial and enduring memory deficit of the Halo group compared to the Ctrl group. In addition, there was a main effect of time $\left(F_{(1,46)}=6.317, P=0.016\right)$, indicating a difference in freezing between recent and remote memory tests, but there was no interaction effect $\left(F_{(1,46)}=0.266, P=\right.$ $0.608)$. Further minute-by-minute data of freezing are shown in Figure 1C. These results suggest that the dHPC-to-RSC neural pathway plays a critical role in acquisition of contextual fear memory. Note that Figure 1B,C combined two data sets $(n=24$ per data set, $n=12$ per group; see Materials and Methods for details and Supplemental Fig. 3 for data/statistics).

On the day of training (memory acquisition; day 0), we also analyzed freezing and, additionally, motion index to determine if the laser stimulation impacted locomotion. We found that both freezing during the total training session $\left(t_{(46)}=-0.091, P=\right.$ 0.928; unpaired $t$-test; Supplemental Fig. 4A, top left) and motion index during the first 2 min before footshocks $\left(t_{(46)}=-0.207, P=\right.$ 0.837; Supplemental Fig. 4A, top right) did not differ between the Halo and Ctrl groups. This suggests that the laser stimulation did not affect freezing expression or general locomotion. After completion of the above experiments, histological verification of optical fiber placements and viral injections revealed that all optical fiber implants were chronically placed around the midline and dorsal to the RSC (Fig. 1D; Supplemental Fig. 1B) and that all dHPC expressed fluorescence. Therefore, all animals were included for analyses.

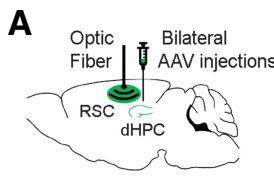

B

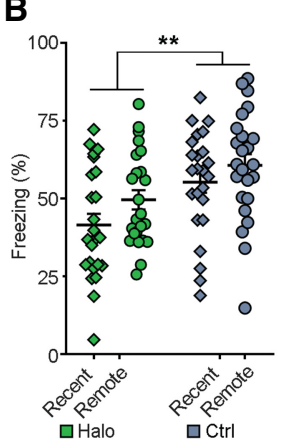

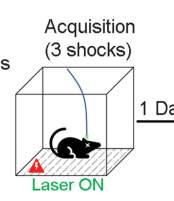
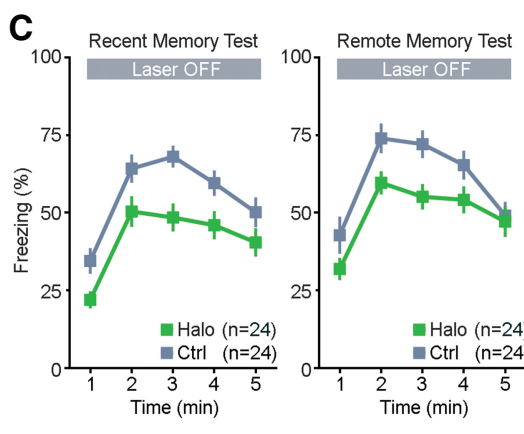

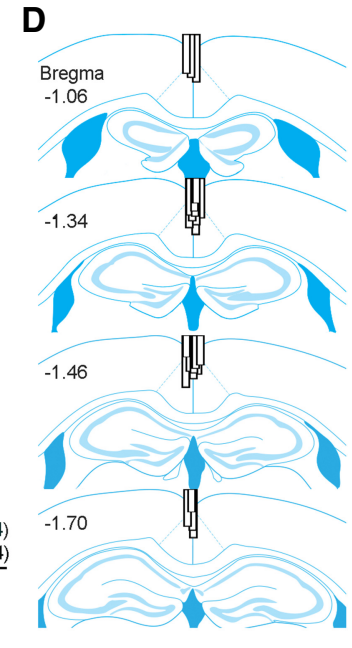

Figure 1. Optogenetic inhibition of dHPC-to-RSC pathway during memory acquisition. $(A)$ Schematic drawing (left) of bilateral injections of AAV viruses that encode either halorhodopsin (Halo) or YFP (Ctrl) into the dHPC and an optical fiber implant into the RSC. Contextual fear conditioning procedure (right). Mice received optogenetic inhibition of the dHPC-to-RSC neuronal terminals during acquisition of memory on day 0 . Then mice were tested for recent memory on day 1 and remote memory on day 31, receiving no laser stimulation. $(B)$ Percentage of freezing (mean of 5 min) during the recent memory test (day 1 ) and remote memory test (day 31 ). $\left(^{* *}\right) P=0.004$, repeated measures ANOVA between groups. (C) Minute-by-minute percentage of freezing from $B$ of recent (left) and remote (right) memory tests. Both left and right, $P<0.05$; Bonferroni post-hoc of the means between Halo/ Ctrl. All error bars indicate standard error of the mean (SEM). (D) Optical fiber placements of the Halo group were confirmed mostly dorsal to the RSC, arranged anterior (top) to posterior (bottom). Adapted from Franklin and Paxinos 2008.

\section{Optogenetic inhibition of dHPC-to-RSC pathway during} memory retrieval

Similarly, mice received bilateral dHPC injections of either AAV-Halo or AAVeYFP and a RSC optical fiber implantation (Fig. 2A, left). Four-five weeks after surgery, mice were trained for acquisition of contextual fear memory on day 0 and were tested subsequently for recent (day 1) and remote (day 31) memories. Mice received photoinhibition of the $\mathrm{dHPC}$ to-RSC neuronal terminals during both testing days (Fig. 2A, right). There was no main effect of group $\left(F_{(1,24)}=\right.$ $0.159, P=0.694$; repeated measures twoway ANOVA; Fig. 2B) or interaction effect $\left(F_{(1,46)}=0.001, P=0.973\right)$, but a main effect of time $\left(F_{(1,24)}=7.053, P=0.014\right)$, indicating a difference in freezing between recent and remote memory tests. Further minute-by-minute data of freezing are shown in Figure 2C. In addition, there was no significant difference in mean freezing between Halo and Ctrl viral groups during acquisition (day $0 ; t_{(46)}=$ $0.648, P=0.523$; unpaired $t$-test; Supplemental Fig. 4B, top). These results suggest 
A
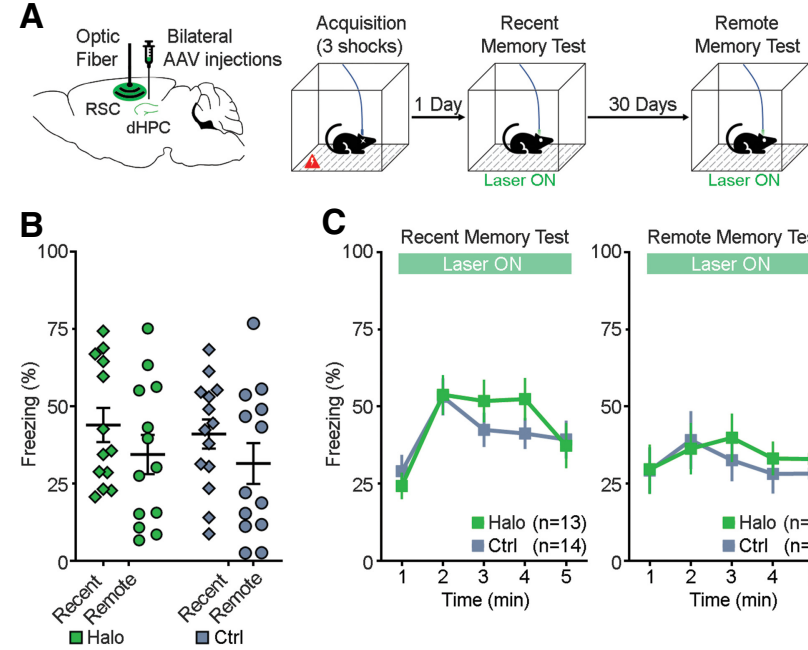

C
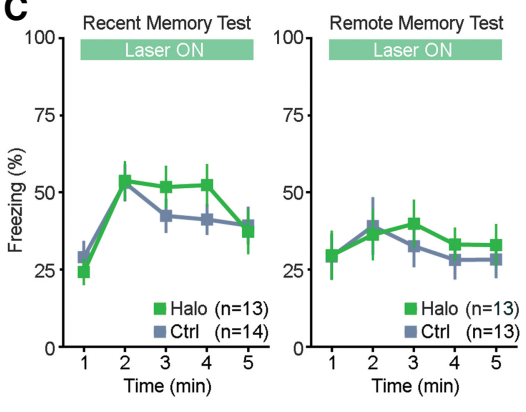

D

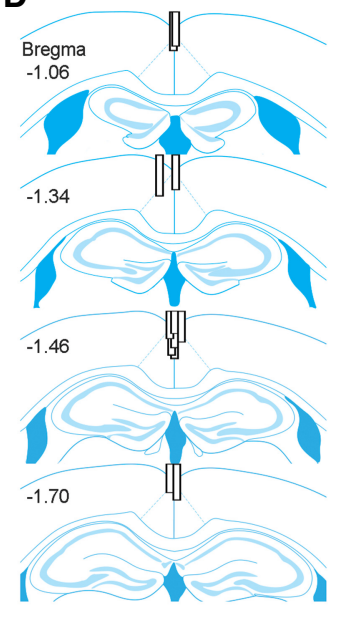

Figure 2. Optogenetic inhibition of dHPC-to-RSC pathway during memory retrieval. (A) Schematic drawing (left) of bilateral injections of AAV viruses that encode either halorhodopsin (Halo) or YFP (Ctrl) into the dHPC and an optical fiber implant into the RSC. Contextual fear conditioning procedure (right). Mice were trained during acquisition of memory on day 0 , receiving no laser stimulation. Then, mice received optogenetic inhibition of the dHPC-to-RSC neuronal terminals during both recent (day 1 ) and remote (day 31) memory tests. (B) Percentage of freezing (mean of $5 \mathrm{~min}$ ) during the recent memory test (day 1 ) and remote memory test (day 31$) . P=0.694$; repeated measures ANOVA between groups. (C) Minute-by-minute percentage of freezing from B of recent (left) and remote (right) memory tests. All error bars indicate SEM. (D) Optical fiber placements of the Halo group were confirmed mostly dorsal to the RSC, arranged anterior (top) to posterior (bottom).

that the dHPC-to-RSC neural pathway is dispensable during the retrieval/expression of contextual fear memory, though we cannot exclude the possibility of an insufficient inhibition in our experiment. After experiments concluded, histological verification revealed that all optical fiber implants were chronically placed near the midline and dorsal to the RSC (Fig. 2D; Supplemental Fig. 1B) and that all dHPC expressed fluorescence. All animals were included for analyses except one (Ctrl) that lost its optical fiber implant after the recent memory test and was sacrificed before the remote memory test.

\section{Optogenetic inhibition of dHPC-to-LS pathway during memory acquisition}

Mice received bilateral dHPC injections of AAV-Halo or AAV-eYFP and an optical fiber implant into the midline immediately above the LS (Fig. 3A, left). This implant is capable of bilateral inhibition because the dHPC (our injection site) projects mainly to the midline rostral LS (Supplemental Fig. 1). Four-five weeks after surgery, mice received photoinhibition of the dHPC-to-LS neuronal terminals during acquisition of contextual fear memory on day 0 . Mice were tested subsequently for recent (day 1) and remote (day 31) memories, receiving no photoinhibition during the tests (Fig. 3A, right). There was a significant main effect of viral group $\left(F_{(1,24)}=4.623, P=0.042\right.$; repeated mea-

\section{A}
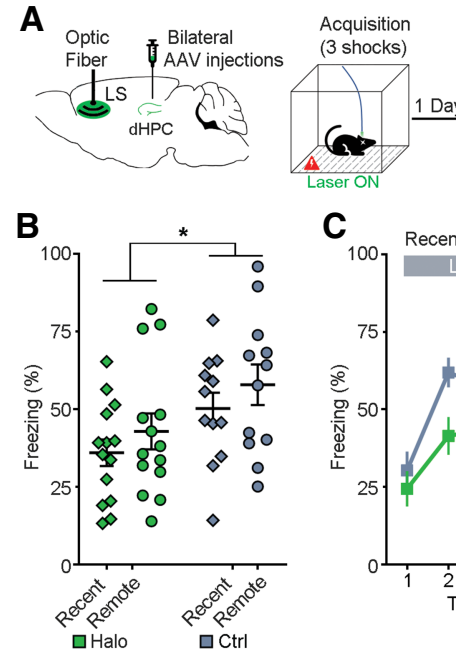

C
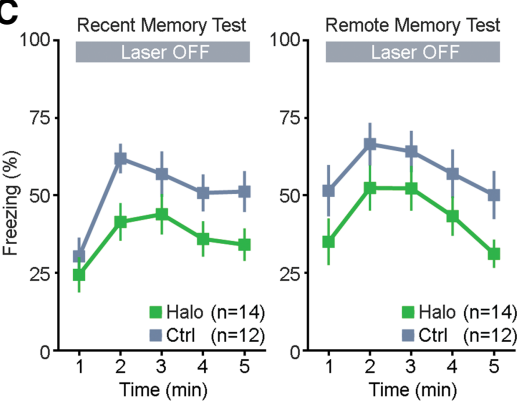

Figure 3. Optogenetic inhibition of dHPC-to-LS pathway during memory acquisition. (A) Schematic drawing (left) of bilateral injections of AAV viruses that encode either halorhodopsin (Halo) or YFP (Ctrl) into the dHPC and an optical fiber implant into the LS. Contextual fear conditioning procedure (right). Mice received optogenetic inhibition of the dHPC-to-LS neuronal terminals during acquisition of memory on day 0 . Then mice were tested for recent memory on day 1 and remote memory on day 31 , receiving no laser stimulation. (B) Percentage of freezing (mean of $5 \mathrm{~min}$ ) during the recent memory test (day 1 ) and remote memory test (day 31 ). ${ }^{*} P=0.042$; repeated measures ANOVA between groups. (C) Minute-by-minute percentage of freezing from $B$ of recent (left) and remote (right) memory tests. Both left and right, $P>0.05$; Bonferroni post-hoc of the means between Halo/ Ctrl. All error bars indicate SEM. (D) Optical fiber placements of the Halo group were confirmed mostly dorsal to the LS, arranged anterior (top) to posterior (bottom). 
A
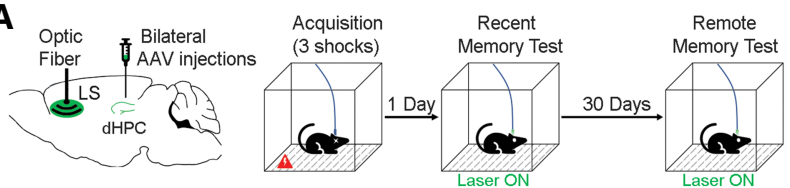

B
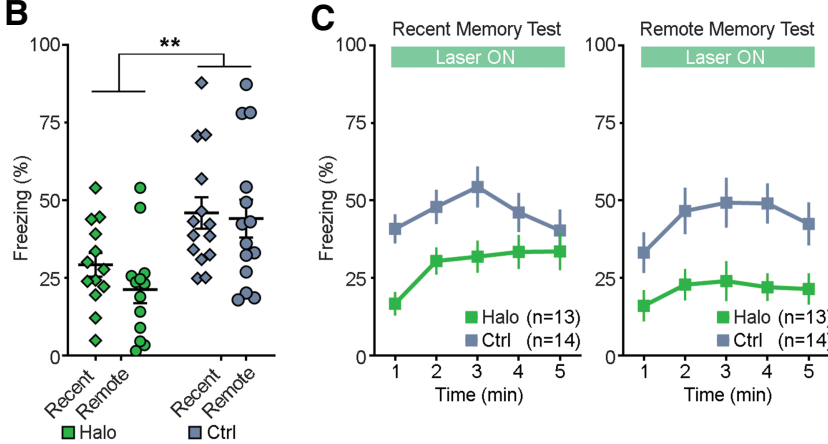

Figure 4. Optogenetic inhibition of dHPC-to-LS pathway during memory retrieval. $(A)$ Schematic drawing (left) of bilateral injections of AAV viruses that encode either halorhodopsin (Halo) or YFP (Ctrl) into the AHPC and an optical fiber implant into the LS. Contextual fear conditioning procedure (right). Mice were trained during acquisition of memory on day 0 , receiving no laser stimulation. Then, mice received optogenetic inhibition of the dHPC-to-LS neuronal terminals during both recent (day 1 ) and remote (day 31 ) memory tests. (B) Percentage of freezing (mean of 5 min) during the recent memory test (day 1 ) and remote memory test (day 31 ). $\left.{ }^{* *}\right) P=0.004$; repeated measures ANOVA between groups. (C) Minute-by-minute percentage of freezing from B of recent (left) and remote (right) memory tests. Both left and right, $P<0.05$; Bonferroni post-hoc of the means between Halo/Ctrl. All error bars indicate SEM. (D) Optical fiber placements of the Halo group were confirmed mostly dorsal to the LS, arranged anterior (top) to posterior (bottom).

that optogenetic inhibition of the dHPC-to-LS pathway has no or limited effect on locomotor activity. After completion of the above experiments, histological verification revealed that all optical fibers were chronically placed around the midline and dorsal to the rostral LS (Fig. 3D; Supplemental Fig. 1C) and that all dHPC expressed fluorescence. Therefore, all animals were included for analyses.
D

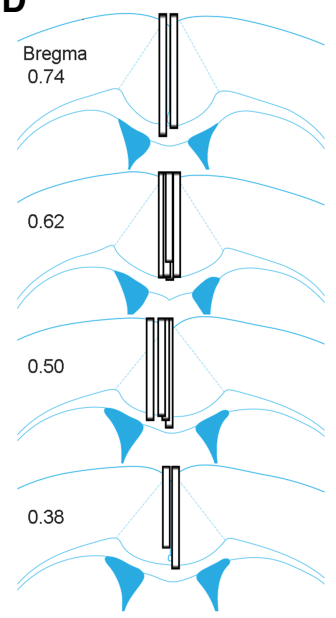

textual fear memory. No significant difference of freezing existed between groups during acquisition of memory (day 0; $t_{(25)}=0.943, P=0.335$; unpaired t-test; Supplemental Fig. 4B, bottom). After experiments concluded, histological verification revealed that all optical fibers were chronically placed on the midline and dorsal to the LS (Fig. 4D; Supplemental Fig. 1C) and that all dHPC expressed fluorescence. Therefore, all animals were included for analyses.

\section{Optogenetic inhibition of dHPC terminals induces firing changes in the RSC and LS}

To confirm that the dHPC projections can influence downstream neural activity, we utilized a combined optogenetic and electrophysiology approach: we unilaterally injected AAV-Halo viruses into the dHPC and implanted an optrode (an optical fiber attached to eight tetrodes) into the ipsilateral granular RSC or LS (Fig. $5 \mathrm{~A}, \mathrm{D})$. About 3 wk after the surgery, we started recording RSC or LS spike activity in freely behaving mice while intermittently delivering photoinhibitions (5 min in every $15 \mathrm{~min} ; \sim 2 \mathrm{~h}$ per session). Our results revealed that photoinhibition of the dHPC projections affected firing of both RSC and LS neurons. A subpopulation of the RSC and LS neurons was silenced or suppressed, demonstrating the effectiveness of our photoinhibition approach (Fig. 5B,C,E,F). Interestingly, another subpopulation of the RSC and LS neurons was activated upon the same photoinhibition (Fig. 5B,C,E,F), which could be explained by a disinhibition mechanism via local interneurons (Opalka et al. 2020)

\section{Optogenetic inhibition of dHPC-to-LS pathway during memory retrieval}

Similarly, mice received bilateral dHPC injections of either AAV-Halo or AAVeYFP and a LS optical fiber implantation (Fig. 4A, left). Four-five weeks after surgery, mice were trained for acquisition of contextual fear memory on day 0 and were tested subsequently for recent memory on day 1 and remote memory on day 31 . Mice received photoinhibition of the dHPC-to-LS neuronal terminals during both testing days (Fig. 4A, right). There was a significant main effect of viral group $\left(F_{(1,25)}=9.818, P=0.004\right.$; repeated measures two-way ANOVA; Fig. 4B), indicating impaired memory of the Halo group compared to the Ctrl group. There was no main effect of time $\left(F_{(1,25)}=2.363, P\right.$ $=0.137)$ or interaction effect $\left(F_{(1,25)}=\right.$ $0.933, P=0.343)$. Further minute-by-minute data of freezing are shown in Figure 4C. These results suggest that the dHPC-to-LS neural pathway plays a critical role in the retrieval/expression of con-
A

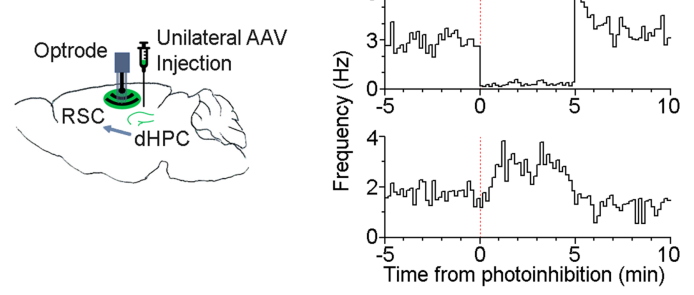

D

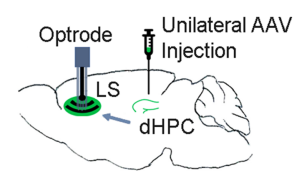

E

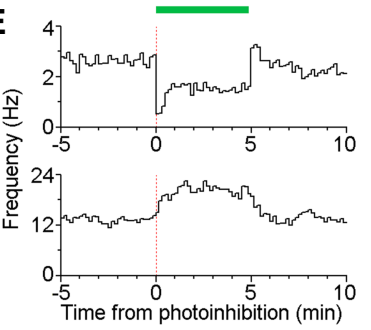

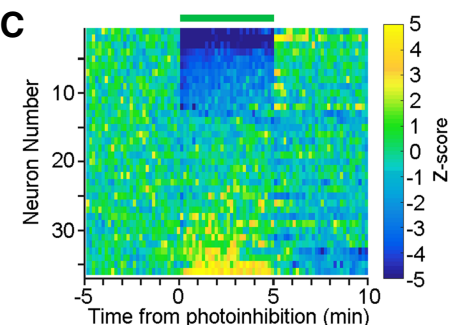

$\mathbf{F}$

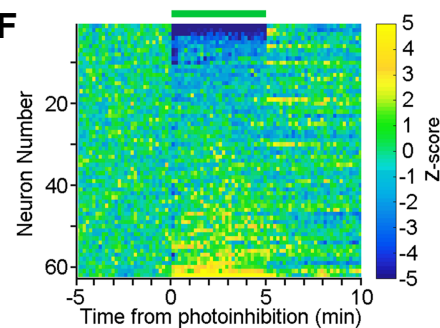

Figure 5. Optogenetic inhibition of dHPC terminals induces RSC and LS firing changes. $(A, D)$ Schematic drawing of unilateral viral injection of AAV-CaMKII-Halo into the dHPC and an optrode implant, an optical fiber attached to eight tetrodes, into either the RSC $(A)$ or LS $(D) .(B, E)$ Two example RSC $(B)$ or LS $(E)$ neurons recorded simultaneously that were inhibited (top) or activated (bottom) upon photoinhibition (green bar, 0-5 min; bin, $10 \mathrm{sec}$ ). (C,F) Heatmap of all RSC (C) and LS $(F)$ neurons upon photoinhibition (green bar, $0-5 \mathrm{~min}$ ). Color bars, $z$-scored firing rate. 
or a network feedback disinhibition. Notably, these results also revealed a functional recovery of the RSC and LS neurons, albeit a small rebound activation in a subset of neurons after the cessation of the photoinhibition (Fig. 5B,C,E,F).

\section{Discussion}

Utilizing an optogenetic approach and contextual fear conditioning paradigm, we provided evidence that the dHPC-to-RSC pathway is critical for memory acquisition, whereas the dHPC-to-LS pathway is critical for both memory acquisition and expression. In both instances, the memory impairments were similar between recent and remote memory tests (one month apart), indicating the reliability of optogenetic manipulation on memory acquisition and expression. Notably, this optogenetic approach enabled transient and reversible inhibition of hippocampal efferents compared to lesions or drug infusions that often cause permanent damages or long-lasting neural alterations. Additionally, our use of the CaMKII promotor likely targeted hippocampal pyramidal neurons but not inhibitory neurons (Liu and Murray 2012; Stark et al. 2012; Wang et al. 2013; Zhu et al. 2014; Besnard et al. 2020); although, future immunostaining or in situ hybridization characterizations are needed to confirm the specificity.

With our optogenetic approach, there was spread of AAV viruses to unintended brain regions. Inside the dHPC, AAV viruses mainly infected the medial CA1 and dorsal subiculum. In some cases, the virus spread partially to the DG, but minimally to the CA3. Given that the DG does not project to the RSC or LS (Van Groen and Wyss 1990; Risold and Swanson 1997; Witter 2007), our observed memory deficits should be mainly attributed to the inactivation of dorsal CA1 and subicular efferents. It should be noted that the LS receives a dense projection from both the CA1 and subiculum, whereas the RSC receives mainly subicular projection (Wyss and Van Groen 1992; Oh et al. 2014; Opalka et al. 2020). Outside of the dHPC, AAV viruses spread mainly along the injection needle track, including the somatosensory and visual anterior area of parietal cortices. Those regions do not project to the LS, although part of the visual area projects to RSC and could be partially responsible for contextual learning. We also noticed some AAV spread to the ventral RSC close to the corpus callosum (Supplemental Fig. 2B), which is unexpected given its long distance from the needle track. Nonetheless, neurons in this ventral RSC were sparsely labeled and, thus, were unlikely to be responsible for the observed memory deficits.

\section{dHPC-to-RSC pathway is critical for memory acquisition}

Previous studies have shown that the RSC plays a critical role during acquisition of multiple forms of memories, including acquisition of contextual fear memory (Corcoran et al. 2011; Cowansage et al. 2014; Kwapis et al. 2015; Todd et al. 2016; de Sousa et al. 2019), inhibitory avoidance memory (Katche and Medina 2017) and trace fear memory (Kwapis et al. 2015). Moreover, using chemogenetic methods, two recent studies found that the dHPC-to-RSC excitatory and inhibitory projections play opposing roles in facilitating and inhibiting memory acquisition, respectively (Yamawaki et al. 2019a,b). Consistently, by using optogenetic methods, we provided evidence that the dHPC-to-RSC projection plays a critical role during memory acquisition. In contrast, the entorhinal-to-RSC pathway appears dispensable during memory acquisition (Kitamura et al. 2017), indicating a functional differentiation of RSC connections. Together, our results add to the growing evidence that hippocampal-cortical communication during memory acquisition is critical for the formation of both recent and remote memories (Kitamura et al. 2017; DeNardo et al. 2019).
Correspondingly, the RSC has been shown to play a critical role during retrieval/expression of multiple forms of memories, including contextual fear memory (Corcoran et al. 2011; Cowansage et al. 2014; Kwapis et al. 2015; Todd et al. 2016), inhibitory avoidance memory (Katche et al. 2013), cued fear memory (Todd et al. 2016), and autobiographical memory (Svoboda et al. 2006). However, it remains unclear which upstream regions control the RSC and support memory retrieval/expression, given that the RSC receives direct inputs from a number of memory associated regions, such as the dHPC, thalamus and cingulate or motor cortices (Oh et al. 2014; Todd et al. 2016). A recent study reported that chemogenetic inhibition of dHPC glutamatergic (VGLUT1) terminals in the RSC impaired the expression of recent but not remote memory (Yamawaki et al. 2019a), whereas our optogenetic inhibition of the dHPC-to-RSC pathway had little effect on the expression of recent or remote memories. One justification for this discrepancy could be due to technical differences, such as spatial restriction and temporal sensitivity of the optogenetic approach compared to chemogenetics. This discrepancy may also be due to different volume viral injections: we injected $0.2 \mu \mathrm{L}$ per hemisphere, limiting viral expression primarily to the medial (distal) CA1 and rostral-dorsal subiculum, whereas their larger injection of $0.5 \mu \mathrm{L}$ per hemisphere likely infected a broader dHPC area that includes the lateral (proximal) CA1 and caudal subiculum. These alternative lateral CA1 and caudal subiculum efferents may support memory retrieval, whereas the medial CA1 and rostral subiculum efferents may support memory acquisition (Nakazawa et al. 2016; Roy et al. 2017).

We noticed that the freezing levels were relatively low during photoinhibition of the dHPC-to-RSC pathway (Fig. 2). However, average freezing still reached $\sim 50 \%$ between $2-4$ min (Fig. $2 \mathrm{C}$, left panel), whereas the baseline freezing level was close to $0 \%$ before training (Supplemental Fig. 4), indicating room for reduction between groups. Therefore, a floor effect was unlikely during the recent memory test (Fig. 2C, left panel); although, there could be a potential floor effect during the remote memory test (Fig. 2C, right panel). Another caveat regards laser stimulation in the RSC potentially penetrating the dHPC region, given the proximity of these two regions. To rule out this possibility, we included a second group of mice that received a lower power photoinhibition $(\sim 0.3$ $\mathrm{mW}$; see Materials and Methods) during acquisition, which revealed a significant difference in freezing between the Halo and Ctrl mice $\left(F_{(1,22)}=7.342, P=0.013\right.$; repeated measures two-way ANOVA). This power of $0.3 \mathrm{~mW}$ is very low, which most likely only inhibited the dHPC terminals in the RSC, further confirming the function of this dHPC-to-RSC communication in memory acquisition.

\section{dHPC-to-LS pathway is critical for memory acquisition and expression}

We found that transient optogenetic inhibition of dHPC projections to the LS during contextual fear acquisition hindered subsequent memory performances, indicating the importance of the dHPC-to-LS pathway in memory acquisition. This is consistent with a previous study showing that inactivation of the LS impaired contextual fear acquisition (Calandreau et al. 2007). Additionally, we found that optogenetic inhibition of dHPC projections to the LS during contextual fear retrieval disrupted fear memory performance, indicating the importance of this dHPC-to-LS pathway in memory expression as well, corroborating with another earlier study (Olton et al. 1978). Together, our results suggest that the dHPC-to-LS pathway is critical for both memory acquisition and expression. In support of this, a previous study showed that transected hippocampal efferents to the subcortex impaired both the acquisition and expression of memory (Hunsaker et al. 2009). 
A recent study reported that the dHPC CA3-to-LS pathway is critical for context discrimination but not retrieval (Besnard et al. 2020), while we found that the dHPC (CA1 and subiculum)-to-LS pathway was critical for context retrieval. Anatomically, the dorsal CA1 and CA3 project to the midline and dorsolateral LS, respectively (Oh et al. 2014). Together, these results suggest that the CA1-to-LS (midline) and CA3-to-LS (dorsolateral) pathways play differential roles, namely, memory retrieval (recalling more generalized context information) and memory discrimination (recalling more detailed context information), respectively. How the downstream regions may integrate this context information would be important for future investigation.

One caveat regards the potential photoinhibition of LS passing fibers (from the dHPC) to additional downstream subcortical regions, such as the diagonal band of Broca and mammillary regions. Future experiments that specifically target these dHPC projections are needed to understand their functions. Additionally, our observed deficit during memory expression may be confounded by reduced freezing expression because the dHPC-to-LS and other LS input pathways have been implicated in processing animal's locomotor speed (Bender et al. 2015; Wirtshafter and Wilson 2019), despite that manipulating the CA1 itself does not affect locomotion (Goshen et al. 2011). In our experiments, inhibition of the dHPC-to-LS pathway did not affect freezing during training but slightly increased locomotion (Supplemental Fig. 4). Therefore, additional experiments that do not rely on freezing as a measurement of memory are necessary to validate the functional role of this dHPC-to-LS pathway in memory retrieval/expression.

\section{Recent and remote memories}

Our results showed that memory impairments were similar between recent and remote memory tests (one month apart). Notably, there was a slight increase of freezing between recent and remote memory tests for mice without optical fiber attachment (inhibition during acquisition; Figs. 1B, 3B). This is consistent with previous studies that reported strengthened remote memory compared to recent memory, which can be explained by a reconsolidation mechanism that enhances memories after retrieval (Fukushima et al. 2014). On the other hand, there was a decrease of freezing between recent and remote memory tests for mice that had an optical fiber attachment (inhibition during retrieval; Figs. 2B, 4B). This reattachment of the optical fiber during the remote memory tests may have distracted the mice and affected their expression of freezing, given that the mice had not experienced the fiber attachment for one month. Furthermore, it is unlikely that the laser leakage from the optical fiber distracted the mice because the optical fiber attachment was covered by a tight sleeve to minimize laser leakage. Additionally, house lights were on during the entire test duration, so the chamber environment was lightened to further minimize any distraction from laser leakage. Nonetheless, the fiber attachment seemed to have little effect during the recent memory tests because animals were habituated to the fiber attachment $24 \mathrm{~h}$ prior to the test.

Together, our study along with others reveal the intricate functional selectivity of memory pathways and provide evidence that multiple memory systems, involving select cortical and subcortical regions during varying stages of memory, may activate in parallel or, conversely, compete under certain circumstances. The dHPC efferents to both cortical (RSC) and subcortical (LS) regions play an important role in memory acquisition. This similar role of the dHPC-to-RSC and dHPC-to-LS projections in fear memory acquisition suggests that the two parallel pathways may contribute to the same or represent different aspects of the fear memory, such as freezing, stress hormones and blood pressure (Roy et al. 2017). Disruption of these pathways during acquisition may also prevent subsequent consolidation of the memory, resulting in the observed memory deficits. On the other hand, the differential roles of the dHPC-to-RSC and dHPC-to-LS pathways involved in fear memory expression reveal either functional disassociation or compensation of multiple memory pathways. These findings convey the importance of further exploration on the neural mechanisms of these pathways to better understand functional neuroanatomy for potential clinical application.

\section{Materials and Methods}

\section{Mice}

Male C57BL/6J mice $(n=131 ; 25-32 \mathrm{~g} ; 10-13$ wk old at the time of surgery) purchased from Jackson Laboratories were used for behavioral tests. After surgery, mice were dually housed (except for optrode mice that were singly housed; Fig. 5) in standard mouse cages $(25 \times 15 \times 15 \mathrm{~cm})$ that contained bedding and environmental enrichment (cotton and wood sticks) on a $12 \mathrm{~h}$ light-dark cycle with ad libitum access to water and food. Mice were removed from the study if the optical fiber headcap fell off before or during experiments $(n=3)$. All experimental procedures were approved by and in accordance with the Institutional Animal Care and Use Committee at Drexel University College of Medicine.

\section{Viral vectors}

Addgene or the University of Pennsylvania Penn Vector Core produced the adeno-associated virus serotype-1 (AAV1) encoding halorhodopsin (eNpHR3.0), enhanced yellow fluorescent protein (eYFP) or enhanced green fluorescent protein (eGFP): AAV1. CaMKIIa.eNpHR3.0.eYFP.WPRE.hGH (Addgene, \#26971), AAV1. CaMKII.eYFP. WPRE.hGH (Addgene, \#105622), AAV1.Syn.eGFP. WPRE.bGH (Penn Vector Core, \#CS1221), respectively. The final viral concentrations were $2.66 \times 10^{13} \mathrm{GC}$ (genome copies) $/ \mathrm{mL}$, $1.00 \times 10^{13} \mathrm{GC} / \mathrm{mL}$ and $2.80 \times 10^{13} \mathrm{GC} / \mathrm{mL}$, respectively.

\section{Stereotaxic surgery}

For optogenetic experiments, pairs of mice were randomly assigned to either experimental (Halo) or control (Ctrl) groups and received the corresponding bilateral AAV viral injections into the dHPC $(0.2 \mu \mathrm{L}$ per injection site; Halo group: AAV1.CaMKII. eNpHR3.0.eYFP, $n=64$; Ctrl group: AAV1.CaMKII.eYFP, $n=22$; or AAV1.Syn.eGFP, $n=42)$. Mice were anesthetized by ketamine/xylazine solution ( 100/10 mg/kg, i.p., Vedco, Inc.) and placed into a Kopf stereotaxic instrument. Breathing was monitored throughout the duration of the surgery to ensure that anesthesia was maintained. After leveling Bregma with Lambda, three 340- $\mu$ m holes were drilled: two bilaterally above the dHPC and one above the midline of either the RSC or LS, depending on experimental group. After drilling, viruses $(200 \mathrm{~nL})$ were microinjected into the dHPC by a syringe pump (World Precision Instruments, WPI) over $4 \mathrm{~min}(50 \mathrm{~nL} / \mathrm{min})$, with an addition of $5 \mathrm{~min}$ before removal of the injection needle (34 gauge, beveled; WPI). The coordinates for the dHPC viral injections were AP $-2.0 \mathrm{~mm}, \mathrm{ML} \pm 0.9 \mathrm{~mm}$, DV $-1.6 \mathrm{~mm}$. Mice also received an optical fiber (200- $\mu \mathrm{m}$ diameter; ThorLabs, Inc.) implanted into either the RSC or LS that was secured with biocompatible ionomer (DenMat Geristore). The coordinates for the RSC optical fiber placement were AP $-1.5 \mathrm{~mm}, \mathrm{ML}$ $0.0 \mathrm{~mm}, \mathrm{DV}-0.7 \mathrm{~mm}$, while the coordinates for the LS optical fiber placement were AP $0.6 \mathrm{~mm}, \mathrm{ML} 0.0 \mathrm{~mm}, \mathrm{DV}-2.05 \mathrm{~mm}$. Behavioral experiments occurred 4-5 wk after surgery to allow gene expression.

For optogenetics combined with electrophysiology recording, an additional four mice were used. These mice received AAV injections into the dHPC (0.2 $\mu \mathrm{L}$; AAV1.CaMKII.eNpHR3.0.eYFP) with the same dHPC coordinates as above. Mice underwent a similar surgical procedure as above, except an optrode, an optical fiber attached to a bundle of eight tetrodes, was implanted into either the RSC $(n=2)$ or LS $(n=2)$. This optrode was coupled with a microdrive to gradually drive the electrodes to deeper recording sites 
post-surgery, as seen in our previous publications (Wang et al. 2015; Opalka et al. 2020). The coordinates for the RSC and LS were slightly adjusted more laterally in order to record cell bodies: the coordinates for the RSC optrode placement were AP $-1.5 \mathrm{~mm}$, ML $0.3 \mathrm{~mm}$, DV -0.9 , while the LS optrode placement were AP 0.6 $\mathrm{mm}, \mathrm{ML} 0.2, \mathrm{DV}-2.0 \mathrm{~mm}$. Recording occurred at least $3 \mathrm{wk}$ after surgery to allow gene expression of eNpHR3.0 on hippocampal terminals.

\section{Experimental design}

Four-five weeks after surgery, mice received $2 \mathrm{~d}$ of handling, 10-15 min on the first day and $\sim 5$ min on the second day. After handling, mice were trained with a contextual fear conditioning procedure in four identical footshock chambers $(32 \times 25 \times 25 \mathrm{~cm})$ illuminated by lights inside sound-attenuating cubicles $(64 \times 75 \times 36 \mathrm{~cm}$; Med Associates, Inc.), silencing the dHPC projection either during acquisition or retrieval/expression of recent and remote contextual memory. To minimize laser leaking from the optical fiber attachment, a tight sleeve (black shrinking tube) covered this attachment. To reduce the mouse's time spent in the footshock chamber before recording began, two chambers were used when one experimenter was present, or four chambers were used when two experimenters were present. All behavioral procedures began around 4:00 P.M. During acquisition and retrieval tests, mice were counterbalanced among pairs and tested in separate chambers. After each mouse was tested, the chamber was cleaned with $70 \%$ ethanol.

\section{Optogenetic inhibition during contextual fear acquisition} During acquisition (day 0), the optical fiber implant of each mouse was connected to a 532 nanometer $(\mathrm{nm})$ green laser (Opto Engine LLC). Then, mice were placed into the footshock chamber (Med Associates, Inc.) and allowed to explore for a total of 270 seconds (s), receiving laser stimulation throughout the entire duration. Three footshocks ( $2 \mathrm{sec}, 0.75 \mathrm{~mA}$ ) were delivered at 120, 180, and $240 \mathrm{sec}$. After acquisition, mice were tested for recent memory the next day (day 1) and remote memory in a month (day 31). Each memory test included a $300 \mathrm{~s}$ exploration period in the same footshock chamber as day 0 but with no laser stimulation. Videos of behavior and freezing scores were collected utilizing video-tracking software (VideoFreeze; Med Associates, Inc.) to determine freezing durations among groups. For laser stimulation, we used a power of $\sim 6$ milliwatts $(\mathrm{mW})$ throughout experiments except for a subset of mice used in Figure 1 in which two RSC data sets of mice were tested ( 24 per set; $n=12$ per group). The first set received $\sim 6 \mathrm{~mW}$ laser stimulation, whereas the second set received $\sim 0.3 \mathrm{~mW}$ laser stimulation (to minimize any leakage of laser into hippocampal regions given the proximity of the RSC and $\mathrm{dHPC}$ ). Despite the large difference of the two laser powers used, the results from the two groups were similar (Supplemental Fig. 3); thus, we combined the two data sets for analysis (Fig. 1).

\section{Optogenetic inhibition during contextual fear retrieval}

During acquisition (day 0), the optical fiber implant of each mouse was connected to a $532 \mathrm{~nm}$ green laser. Then, mice were placed into the footshock chamber (Med Associates, Inc.) and allowed to explore for a total of $270 \mathrm{sec}$, receiving no laser stimulation. Three footshocks ( $2 \mathrm{sec}, 0.75 \mathrm{~mA}$ ) were delivered at 120, 180, and $240 \mathrm{sec}$. After acquisition, mice were tested for recent memory on the next day (day 1) and remote memory in a month (day 31). For each memory test, the optical fiber implant of each mouse was connected to a $532 \mathrm{~nm}$ green laser. Mice were then placed in the same footshock chamber as day 0 for a total of $480 \mathrm{sec}$, receiving laser stimulation for the first $300 \mathrm{sec}$. Data from 300-480 sec are shown in Supplemental Figure 5. Videos of behavior and freezing scores were collected utilizing video-tracking software (VideoFreeze; Med Associates, Inc.) to determine freezing durations among groups. For laser stimulation, we used a power of $\sim 6 \mathrm{~mW}$ throughout.

\section{Data analysis}

For behavioral experiments without the optical fiber connected to the mouse (contextual fear acquisition memory tests), freezing was calculated using the VideoFreeze software (motion index $\leq 18$ and lasted for at least $1 \mathrm{sec}$ was considered freezing) (Anagnostaras et al. 2010; Wang et al. 2015). Freezing scores were exported from Video Freeze, and then percentage of freezing per minute was further calculated in MATLAB. For behavioral experiments with the optical fiber connected to the mouse (all training/acquisition and contextual fear retrieval memory tests), we noticed that sometimes mice were visibly freezing, but the VideoFreeze software calculated swaying of the optical fiber tether as mouse movement, rendering the freezing calculation inaccurate. Thus, we reduced this fiber movement effect by cropping the top $75 \%$ of video frames that almost exclusively recorded fiber but not mouse movement (mice were typically recorded in the bottom $25 \%$ of video frames by a side-view camera except during footshocks). We then used MATLAB to analyze the cut video (bottom 25\%) and calculate mouse freezing index with the same parameters as VideoFreeze (motion index $\leq 18$ and lasted for at least $1 \mathrm{sec}$ ). Repeated measures two-way ANOVAs and Bonferroni post-hoc tests were used for analyses. All statistics were run in SPSS (IBM). All mice were included in the analysis $(n=128)$ except those with their headcap detached before or during experiments $(n=3)$.

\section{In vivo electrophysiology}

We used optrodes, optic fibers attached to eight tetrodes for recording, similar to that shown previously (Opalka et al. 2020). Each tetrode consisted of eight wires (90\% platinum and 10\% iridium; $18 \mu \mathrm{m}$ diameter; California Fine Wire). About $3 \mathrm{wk}$ after surgery, tetrodes were screened for spike activity. Neural signals were preamplified, digitized, and recorded using a Neuralynx Digital Lynx acquisition system. Each electrode bundle was lowered by 50-100 $\mu \mathrm{m}$ daily until multiple RSC or LS neurons were detected. Spikes were digitized at $30 \mathrm{kHz}$ and filtered at $600-6000 \mathrm{~Hz}$, using ground as the reference for both. All mice received two to five sessions of recording when they were freely behaving or sleeping in home cages $(\sim 2 \mathrm{~h})$. After completion of each recording session, the electrode bundle was lowered by $50-100 \mu \mathrm{m}$ to record a deeper site in the RSC or LS: a total of two to five sites were recorded from each mouse. Five-minute continuous laser stimulations $(\sim 6 \mathrm{~mW}$; $532 \mathrm{~nm}$; Opto Engine LLC) were used in each session $(\sim 2 \mathrm{~h})$, with an intertrial interval of $15 \mathrm{~min}$.

\section{Histology}

After completion of remote memory tests (or locomotor tests when applicable), mice received pentobarbital overdose and were perfused transcardially with $1 \times$ PBS for $\sim 4$ min followed by $10 \%$ formalin at a rate of $1.3 \mathrm{~mL} / \mathrm{min}$ until muscle contractions occurred. Then, $10 \%$ formalin was perfused for $30 \mathrm{~min}$ at a rate of $0.3 \mathrm{~mL} / \mathrm{min}$. Brains were stored in $10 \%$ formalin for at least $24 \mathrm{~h}$ before vibratome sectioning (Leica VT1000 S). Coronal sections $(50 \mu \mathrm{m})$ were collected to verify all injection sites, viral expression, and optical fiber placements.

\section{Competing interest statement}

The authors declare no competing interests.

\section{Acknowledgments}

We thank the Histology and Imaging Facilities at Drexel University Department of Neurobiology and Anatomy for their generous help. We also thank Dr. Jed Shumsky and Dr. Rodrigo España for their advice on statistics and Candace Rizzi-Wise for her editing of our manuscript. This research was supported by Drexel University College of Medicine and NIMH/NIH (R01 MH119102).

Author contributions: A.N.O. and D.V.W. designed and conducted the experiments, analyzed the data, and wrote the manuscript. 


\section{References}

Anagnostaras S, Wood S, Shuman T, Cai D, LeDuc A, Zurn K, Zurn JB, Sage J, Herrera G. 2010. Automated assessment of Pavlovian conditioned freezing and shock reactivity in mice using the video freeze system. Front Behav Neurosci 4: 158. doi:10.3389/fnbeh.2010.00158

Bender F, Gorbati M, Cadavieco MC, Denisova N, Gao X, Holman C, Korotkova T, Ponomarenko A. 2015. Theta oscillations regulate the speed of locomotion via a hippocampus to lateral septum pathway. Nat Commun 6: 8521. doi:10.1038/ncomms9521

Besnard A, Gao Y, TaeWoo Kim M, Twarkowski H, Reed AK, Langberg T, Feng W, Xu X, Saur D, Zweifel LS, et al. 2019. Dorsolateral septum somatostatin interneurons gate mobility to calibrate context-specific behavioral fear responses. Nat Neurosci 22: 436-446. doi:10.1038/ s41593-018-0330-y

Besnard A, Miller SM, Sahay A. 2020. Distinct dorsal and ventral hippocampal CA3 outputs govern contextual fear discrimination. Cell Rep 30: 2360-2373 e2365. doi:10.1016/j.celrep.2020.01.055

Calandreau L, Jaffard R, Desmedt A. 2007. Dissociated roles for the lateral and medial septum in elemental and contextual fear conditioning. Learn Mem 14: 422-429. doi:10.1101/lm.531407

Calandreau L, Desgranges B, Jaffard R, Desmedt A. 2010. Switching from contextual to tone fear conditioning and vice versa: the key role of the glutamatergic hippocampal-lateral septal neurotransmission. Learn Mem 17: 440-443. doi:10.1101/lm.1859810

Carr MF, Jadhav SP, Frank LM. 2011. Hippocampal replay in the awake state: a potential substrate for memory consolidation and retrieval. Nat Neurosci 14: 147 . doi:10.1038/nn.2732

Colgin LL. 2016. Rhythms of the hippocampal network. Nat Rev Neurosci 17: 239. doi:10.1038/nrn.2016.21

Cooper BG, Manka TF, Mizumori SJ. 2001. Finding your way in the dark: the retrosplenial cortex contributes to spatial memory and navigation without visual cues. Behav Neurosci 115: 1012-1028. doi:10.1037/ 0735-7044.115.5.1012

Corcoran KA, Donnan MD, Tronson NC, Guzmán YF, Gao C, Jovasevic V, Guedea AL, Radulovic J. 2011. NMDA receptors in retrosplenial cortex are necessary for retrieval of recent and remote context fear memory. $J$ Neurosci 31: 11655-11659. doi:10.1523/JNEUROSCI.2107-11.2011

Cowansage KK, Shuman T, Dillingham BC, Chang A, Golshani P, Mayford M. 2014. Direct Reactivation of a Coherent Neocortical Memory of Context. Neuron 84: 432-441. doi:10.1016/j.neuron.2014 .09 .022

Czajkowski R, Jayaprakash B, Wiltgen B, Rogerson T, Guzman-Karlsson MC, Barth AL, Trachtenberg JT, Silva AJ. 2014. Encoding and storage of spatial information in the retrosplenial cortex. Proc Natl Acad Sci 111: 8661-8666. doi:10.1073/pnas.1313222111

Dantzer R, Koob GF, Bluthé R-M, Le Moal M. 1988. Septal vasopressin modulates social memory in male rats. Brain Res 457: 143-147. doi:10 .1016/0006-8993(88)90066-2

DeNardo LA, Liu CD, Allen WE, Adams EL, Friedmann D, Fu L, Guenthner CJ, Tessier-Lavigne M, Luo L. 2019. Temporal evolution of cortical ensembles promoting remote memory retrieval. Nat Neurosci 22: 460-469. doi:10.1038/s41593-018-0318-7

de Sousa AF, Cowansage KK, Zutshi I, Cardozo LM, Yoo EJ, Leutgeb S, Mayford M. 2019. Optogenetic reactivation of memory ensembles in the retrosplenial cortex induces systems consolidation. Proc Natl Acad Sci 116: 8576-8581. doi:10.1073/pnas.1818432116

Everts HGJ, Koolhaas JM. 1997. Lateral septal vasopressin in rats: role in social and object recognition? Brain Res 760: 1-7. doi:10.1016/ S0006-8993(97)00269-2

Frankland PW, Bontempi B, Talton LE, Kaczmarek L, Silva AJ. 2004. The involvement of the anterior cingulate cortex in remote contextual fear memory. Science 304: 881-883. doi:10.1126/science.1094804

Franklin K, Paxinos G. 2008. The mouse brain in stereotaxic coordinates, Compact 3rd ed. Academic, New York.

Fukushima H, Zhang Y, Archbold G, Ishikawa R, Nader K, Kida S. 2014. Enhancement of fear memory by retrieval through reconsolidation. Elife 3: e02736. doi:10.7554/eLife.02736

Goshen I, Brodsky M, Prakash R, Wallace J, Gradinaru V, Ramakrishnan C, Deisseroth K. 2011. Dynamics of retrieval strategies for remote memories. Cell 147: 678-689. doi:10.1016/j.cell.2011.09.033

Hunsaker MR, Tran GT, Kesner RP. 2009. A behavioral analysis of the role of CA3 and CA1 subcortical efferents during classical fear conditioning. Behav Neurosci 123: 624-630. doi:10.1037/a0015455

Jinno S, Klausberger T, Marton LF, Dalezios Y, Roberts JDB, Fuentealba P, Bushong EA, Henze D, Buzsáki G, Somogyi P. 2007. Neuronal diversity in GABAergic long-range projections from the hippocampus. J Neurosci 27: 8790-8804. doi:10.1523/JNEUROSCI.1847-07.2007

Katche C, Medina JH. 2017. Requirement of an early activation of BDNF/ c-Fos cascade in the retrosplenial cortex for the persistence of a long-lasting aversive memory. Cereb Cortex 27: 1060-1067. doi:10.1093/ cercor/bhv 284
Katche C, Dorman G, Slipczuk L, Cammarota M, Medina JH. 2013. Functional integrity of the retrosplenial cortex is essential for rapid consolidation and recall of fear memory. Learn Mem 20: 170-173. doi:10 $.1101 / \mathrm{lm} .030080 .112$

Keene CS, Bucci DJ. 2008. Contributions of the retrosplenial and posterior parietal cortices to cue-specific and contextual fear conditioning. Behav Neurosci 122: 89-97. doi:10.1037/0735-7044.122.1.89

Kitamura T, Ogawa SK, Roy DS, Okuyama T, Morrissey MD, Smith LM, Redondo RL, Tonegawa S. 2017. Engrams and circuits crucial for systems consolidation of a memory. Science 356: 73. doi:10.1126/science .aam6808

Kwapis JL, Jarome TJ, Lee JL, Helmstetter FJ. 2015. The retrosplenial cortex is involved in the formation of memory for context and trace fear conditioning. Neurobiol Learn Mem 123: 110-116. doi:10.1016/j.nlm .2015.06.007

Lee I, Kesner RP. 2004. Differential contributions of dorsal hippocampal subregions to memory acquisition and retrieval in contextual fear-conditioning. Hippocampus 14: 301-310. doi:10.1002/hipo.10177

Leroy F, Park J, Asok A, Brann DH, Meira T, Boyle LM, Buss EW, Kandel ER, Siegelbaum SA. 2018. A circuit from hippocampal CA2 to lateral septum disinhibits social aggression. Nature 564: 213-218. doi:10.1038/ s41586-018-0772-0

Lesburguères E, Gobbo OL, Alaux-Cantin S, Hambucken A, Trifilieff P, Bontempi B. 2011. Early tagging of cortical networks is required for the formation of enduring associative memory. Science 331: 924 . doi:10 $.1126 /$ science. 1196164

Liu XB, Murray KD. 2012. Neuronal excitability and calcium/ calmodulin-dependent protein kinase type II: location, location, location. Epilepsia 53: 45-52.

Mao D, Kandler S, McNaughton BL, Bonin V. 2017. Sparse orthogonal population representation of spatial context in the retrosplenial cortex. Nat Commun 8: 243. doi:10.1038/s41467-017-00180-9

Maren S, Fanselow MS. 1997. Electrolytic lesions of the fimbria/fornix, dorsal hippocampus, or entorhinal cortex produce anterograde deficits in contextual fear conditioning in rats. Neurobiol Learn Mem 67: 142149. doi:10.1006/nlme.1996.3752

Maviel T, Durkin TP, Menzaghi F, Bontempi B. 2004. Sites of neocortical reorganization critical for remote spatial memory. Science 305: 96. doi:10.1126/science. 1098180

McEchron MD, Bouwmeester H, Tseng W, Weiss C, Disterhoft JF. 1998. Hippocampectomy disrupts auditory trace fear conditioning and contextual fear conditioning in the rat. Hippocampus 8: 638-646. doi:10 .1002/(SICI)1098-1063(1998)8:6<638::AID-HIPO6>3.0.CO;2-Q

McGlinchey EM, Aston-Jones G. 2018. Dorsal hippocampus drives context-induced cocaine seeking via inputs to lateral septum. Neuropsychopharmacology 43: 987-1000. doi:10.1038/npp.2017.144

Milczarek MM, Vann SD, Sengpiel F. 2018. Spatial memory engram in the mouse retrosplenial cortex. Curr Biol 28: 1975-1980 e1976. doi:10.1016/ j.cub.2018.05.002

Miller AMP, Frick BJ, Smith DM, Radulovic J, Corcoran KA. 2017. Network oscillatory activity driven by context memory processing is differently regulated by glutamatergic and cholinergic neurotransmission. Neurobiol Learn Mem 145: 59-66. doi:10.1016/j.nlm.2017.08.010

Misane I, Tovote P, Meyer M, Spiess J, Ögren SO, Stiedl O. 2005. Time-dependent involvement of the dorsal hippocampus in trace fear conditioning in mice. Hippocampus 15: 418-426. doi:10.1002/hipo .20067

Miyashita T, Rockland KS. 2007. GABAergic projections from the hippocampus to the retrosplenial cortex in the rat. Eur J Neurosci 26: 1193-1204. doi:10.1111/j.1460-9568.2007.05745.x

Mondragón-Rodríguez S, Gu N, Fasano C, Peña-Ortega F, Williams S. 2019. Functional connectivity between hippocampus and lateral septum is affected in very young Alzheimer's transgenic mouse model. Neuroscience 401: 96-105. doi:10.1016/j.neuroscience.2018.12.042

Nakazawa Y, Pevzner A, Tanaka KZ, Wiltgen BJ. 2016. Memory retrieval along the proximodistal axis of CA1. Hippocampus 26: 1140-1148. doi:10.1002/hipo. 22596

Nitzan N, McKenzie S, Beed P, English DF, Oldani S, Tukker JJ, Buzsaki G, Schmitz D. 2020. Propagation of hippocampal ripples to the neocortex by way of a subiculum-retrosplenial pathway. Nat Commun 11: 1947. doi:10.1038/s41467-020-15787-8

Ocampo AC, Squire LR, Clark RE. 2017. Hippocampal area CA1 and remote memory in rats. Learn Mem 24: 563-568. doi:10.1101/lm.045781.117

Oh SW, Harris JA, Ng L, Winslow B, Cain N, Mihalas S, Wang Q, Lau C, Kuan L, Henry AM, et al. 2014. A mesoscale connectome of the mouse brain. Nature 508: 207-214. doi:10.1038/nature13186

Olton DS, Walker JA, Gage FH. 1978. Hippocampal connections and spatial discrimination. Brain Res 139: 295-308. doi:10.1016/0006-8993(78) 90930-7

Opalka AN, Huang WQ, Liu J, Liang H, Wang DV. 2020. Hippocampal ripple coordinates retrosplenial inhibitory neurons during slow-wave sleep. Cell Rep 30: 432-441 e433. doi:10.1016/j.celrep.2019.12.038 
Pierson JL, Pullins SE, Quinn JJ. 2015. Dorsal hippocampus infusions of CNQX into the dentate gyrus disrupt expression of trace fear conditioning. Hippocampus 25: 779-785. doi:10.1002/hipo.22413

Risold PY, Swanson LW. 1997. Connections of the rat lateral septal complex. Brain Res Rev 24: 115-195. doi:10.1016/S0165-0173(97)00009-X

Robinson S, Keene CS, Iaccarino HF, Duan D, Bucci DJ. 2011. Involvement of retrosplenial cortex in forming associations between multiple sensory stimuli. Behav Neurosci 125: 578-587. doi:10.1037/a0024262

Robinson S, Todd TP, Pasternak AR, Luikart BW, Skelton PD, Urban DJ, Bucci DJ. 2014. Chemogenetic silencing of neurons in retrosplenial cortex disrupts sensory preconditioning. J Neurosci 34: 10982-10988. doi:10.1523/JNEUROSCI.1349-14.2014

Robinson S, Adelman JS, Mogul AS, Ihle PCJ, Davino GM. 2018. Putting fear in context: elucidating the role of the retrosplenial cortex in context discrimination in rats. Neurobiol Learn Mem 148: 50-59. doi:10.1016/j .nlm.2017.12.009

Roy DS, Kitamura T, Okuyama T, Ogawa SK, Sun C, Obata Y, Yoshiki A, Tonegawa S. 2017. Distinct neural circuits for the formation and retrieval of episodic memories. Cell 170: 1000-1012 e1019. doi:10 $.1016 /$ j.cell.2017.07.013

Scoville WB, Milner B. 1957. Loss of recent memory after bilateral hippocampal lesions. J Neurol Neurosurg Psychiatry 20: 11-21. doi:10 .1136/innp.20.1.11

Stark E, Koos T, Buzsaki G. 2012. Diode probes for spatiotemporal optical control of multiple neurons in freely moving animals. J Neurophysiol 108: $349-363$. doi:10.1152/jn.00153.2012

Svoboda E, McKinnon MC, Levine B. 2006. The functional neuroanatomy of autobiographical memory: a meta-analysis. Neuropsychologia 44: 2189-2208. doi:10.1016/j.neuropsychologia.2006.05.023

Takata N, Yoshida K, Komaki Y, Xu M, Sakai Y, Hikishima K, Mimura M, Okano H, Tanaka KF. 2015. Optogenetic activation of CA1 pyramidal neurons at the dorsal and ventral hippocampus evokes distinct brain-wide responses revealed by mouse fMRI. PLoS One 10: e0121417. doi:10.1371/journal.pone.0121417

Tingley D, Buzsáki G. 2018. Transformation of a spatial map across the hippocampal-lateral septal circuit. Neuron 98: 1229-1242.e1225. doi:10 $.1016 /$ j.neuron.2018.04.028

Todd TP, Bucci DJ. 2015. Retrosplenial cortex and long-term memory: molecules to behavior. Neural Plast 2015: 9. doi:10.1155/2015/414173

Todd TP, Mehlman ML, Keene CS, DeAngeli NE, Bucci DJ. 2016. Retrosplenial cortex is required for the retrieval of remote memory for auditory cues. Learn Mem 23: 278-288. doi:10.1101/lm.041822.116
Van Groen T, Wyss JM. 1990. Extrinsic projections from area CA1 of the rat hippocampus: olfactory, cortical, subcortical, and bilateral hippocampal formation projections. J Comp Neurol 302: 515-528. doi:10.1002/cne 903020308

Vann SD, Aggleton JP. 2002. Extensive cytotoxic lesions of the rat retrosplenial cortex reveal consistent deficits on tasks that tax allocentric spatial memory. Behav Neurosci 116: 85-94. doi:10.1037/0735-7044.116 .1 .85

Wang DV, Ikemoto S. 2016. Coordinated interaction between hippocampal sharp-wave ripples and anterior cingulate unit activity. J Neurosci 36: 10663. doi:10.1523/JNEUROSCI.1042-16.2016

Wang X, Zhang C, Szabo G, Sun QQ. 2013. Distribution of CaMKIIalpha expression in the brain in vivo, studied by CaMKIIalpha-GFP mice. Brain Res 1518: 9-25. doi:10.1016/j.brainres.2013.04.042

Wang DV, Yau HJ, Broker CJ, Tsou JH, Bonci A, Ikemoto S. 2015. Mesopontine median raphe regulates hippocampal ripple oscillation and memory consolidation. Nat Neurosci 18: 728-735. doi:10.1038/nn .3998

Wirtshafter HS, Wilson MA. 2019. Locomotor and hippocampal processing converge in the lateral septum. Curr Biol 29: 3177-3192 e3173. doi:10 $.1016 /$ j.cub.2019.07.089

Witter MP. 2007. Intrinsic and extrinsic wiring of CA3: indications for connectional heterogeneity. Learn Mem 14: 705-713. doi:10.1101/lm .725207

Wyss JM, Van Groen T. 1992. Connections between the retrosplenial cortex and the hippocampal formation in the rat: a review. Hippocampus 2: 111. doi:10.1002/hipo.450020102

Yamawaki N, Corcoran KA, Guedea AL, Shepherd GMG, Radulovic J. 2019a. Differential contributions of glutamatergic hippocampal->retrosplenial cortical projections to the formation and persistence of context memories. Cereb Cortex 29: 2728-2736. doi:10.1093/cercor/bhy142

Yamawaki N, Li X, Lambot L, Ren LY, Radulovic J, Shepherd GMG. 2019b. Long-range inhibitory intersection of a retrosplenial thalamocortical circuit by apical tuft-targeting CA1 neurons. Nat Neurosci 22: 618-626. doi:10.1038/s41593-019-0355-x

Zhu H, Pleil KE, Urban DJ, Moy SS, Kash TL, Roth BL. 2014. Chemogenetic inactivation of ventral hippocampal glutamatergic neurons disrupts consolidation of contextual fear memory. Neuropsychopharmacology 39: 1880-1892. doi:10.1038/npp.2014.35

Received April 9, 2020; accepted in revised form June 10, 2020. 


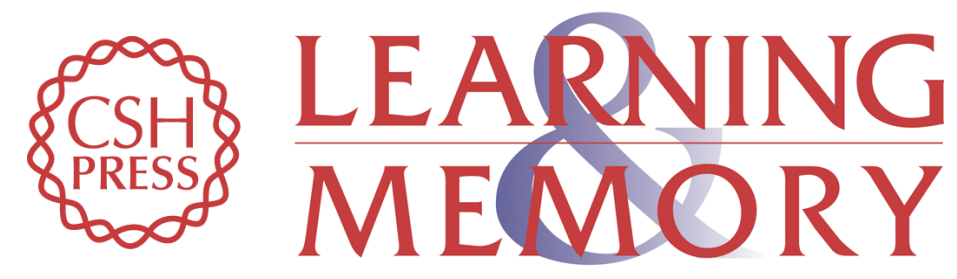

\section{Hippocampal efferents to retrosplenial cortex and lateral septum are required for memory acquisition}

Ashley N. Opalka and Dong V. Wang

Learn. Mem. 2020, 27:

Access the most recent version at doi:10.1101/Im.051797.120

\section{Supplemental http://learnmem.cshlp.org/content/suppl/2020/07/09/27.8.310.DC1 Material}

References This article cites 70 articles, 17 of which can be accessed free at: http://learnmem.cshlp.org/content/27/8/310.full.html\#ref-list-1

Creative This article is distributed exclusively by Cold Spring Harbor Laboratory Press for the Commons first 12 months after the full-issue publication date (see

License http://learnmem.cshlp.org/site/misc/terms.xhtml). After 12 months, it is available under a Creative Commons License (Attribution-NonCommercial 4.0 International), as described at http://creativecommons.org/licenses/by-nc/4.0/.

Email Alerting Receive free email alerts when new articles cite this article - sign up in the box at the Service top right corner of the article or click here. 